\title{
Follow-up of the monitored levels of preservative sensitivity in Europe. Overview of the years 2001-2008
}

\author{
Cecilia Svedman ${ }^{1}$, Klaus E. Andersen², F. M. Brandão³ , Derk P. Bruynzeel ${ }^{4}$, Thomas L. Diepgen ${ }^{5}$, \\ Peter J. Frosch ${ }^{6}$, Thomas Rustemeyer ${ }^{4}$, Ana Giménez-Arnau ${ }^{7}$, Margarida Gonçalo ${ }^{8}$, An Goossens ${ }^{9}$, \\ Jeanne Duus Johansen ${ }^{10}$, Arto Lahti ${ }^{11}$, Torkil Menné ${ }^{10}$, Stefania Seidenari ${ }^{12}$, Antonella Tosti ${ }^{13}$, \\ Jan E. Wahlberg ${ }^{14}$, lan R. White ${ }^{15}$, John D. Wilkinson ${ }^{15}$, Martin Mowitz ${ }^{1}$ and Magnus Bruze ${ }^{1}$
}

${ }^{1}$ Department of Occupational and Environmental Dermatology, SUS, University of Lund, Malmö 20502, Sweden, ${ }^{2}$ Department of Dermatology and Allergy Centre, Odense University Hospital, Odense 5000, Denmark, ${ }^{3}$ Department of Dermatology, Hospital Garcia de Orta, Almada 2805-267, Portugal, ${ }^{4}$ Department of Dermatology, Free University Hospital, Amsterdam NL-1081 HV, The Netherlands, ${ }^{5}$ Department of Social Medicine, Centre of Dermato-Epidemiology, Occupational and Environmental Dermatology, University Hospital Heidelberg, Heidelberg 69115, Germany, ${ }^{6}$ Department of Dermatology, University of Witten/Herdecke, Dortmund 44137, Germany, 7 Department of Dermatology, Hospital del Mar, Universitat Autóma de Barcelona, Barcelona 08003, Spain, ${ }^{8}$ Faculty of Medicine, Department of Dermatology, University Hospital, Coimbra P3000-175, Portugal, ${ }^{9}$ Contact Allergy Unit, Department of Dermatology, U.Z.K.U., Leuven 3000, Belgium, ${ }^{10}$ Department of Dermato-Allergology, National Research Centre, Gentofte Hospital, University of Copenhagen, Hellerup 2900, Denmark, ${ }^{11}$ Department of Dermatology, University of Oulu, Oulu Fl-90014, Finland, 12 Department of Dermatology, University of Modena, Modena 41100, Italy, ${ }^{13}$ Department of Dermatology, University of Bologna, Bologna I-40138, Italy, ${ }^{14}$ Norrbacka, Stockholm 171 76, Sweden, and ${ }^{15}$ Department of Cutaneous Allergy, St John's Institute of Dermatology, London SEI 7EH, UK

doi:10.1111/j.1600-0536.2012.02140.x

Key words: contact allergy; Europe; formaldehyde; formaldehyde-releasers; isothiazolinones; preservatives; trends.

Correspondence: Cecilia Svedman, Department of Occupational and Environmental Dermatology, SUS, University of Lund, Malmö 205 02, Sweden. Tel: +46-40-337813. E-mail: Cecilia.Svedman@skane.se

Conflicts of interest: The authors have declared no conflicts.
The European Environmental and Contact Dermatitis Research Group (EECDRG) previously published the annual levels of contact allergy to preservatives in Europe between the years 1991 and 2000 (1). Since this overview, several reports have described the trends in occurrence of contact allergy to preservatives over the years in Europe (2-4). As trading and industries become 
increasingly globalized, the importance of measuring contact allergy trends on an international basis is increasing $(1,5,6)$. The aim of this study was to present a follow-up of the contact allergy rates for common preservatives in European centres represented by the EECDRG. Fifteen centres, five Nordic, five mid-European, and five southern European, participated, but did not submit data every year; thus, the centres and the number of centres submitting data were not the same every year.

One important reason for monitoring levels of contact allergy is that it allows us to show trends that may otherwise be missed, enabling possible primary, or at least secondary, prevention by legislative measures $(1,5)$.

The different agents used as preservatives in cosmetics, or similar substances, are often also found as biocides in household and industrial products. This means that a contact allergy to a preservative may have both environmental and occupational relevance (1). This is the major reason for five preservatives - parabens, formaldehyde, quaternium-15, methylchloroisothiazolinone (MCI)/methylisothiazolinone) (MI), and methyl dibromo glutaronitrile (MDBGN) - being included in the European baseline series (7).

This report covers the period of 2001-2008, and focuses on the seven most common preservatives that were routinely tested in 12 centres in eight countries. As with many multicentre studies, the limitations of the previous report and the present one include differences between centres regarding the routines for testing, including test materials (Chemotechnique Diagnostics, Trolab ${ }^{\circledR}$, TRUE Test $\left.{ }^{\mathrm{TM}}\right)$, chambers, and readings $(8,9)$. In this study, the number of participating clinics differed between the years, and was not as high as in the previous report (1), in which the number of participating clinics was 16 .

\section{Methods}

Patch testing was based on the International Contact Dermatitis Research Group guidelines. Readings were normally performed on day 2 (or day 3 ) and day 4 , with some centres also re-reading at 6-10 days.

Only the results of those preservatives that were routinely tested as part of the extended European baseline series are reported. These include formaldehyde $1 \%$ aqua, quaternium-15 1\% pet., imidazolidinyl urea $2 \%$ pet., diazolidinyl urea $2 \%$ pet., paraben mix $16 \%$ pet., MCI/MI $0.01 \%$ aqua (from the mid-1980s tested at $0.01 \%$ in Malmö), and MDBGN $0.2-0.5 \%$ pet. (Complete data on preservative sensitivity for quaternium-15 1\% pet., imidazolidinyl urea $2 \%$ pet., diazolidinyl urea $2 \%$ pet., paraben mix $16 \%$ pet. and MDBGN $0.2-0.5 \%$ pet.were not available from every centre for every year.)

\section{Results}

In Fig. 1, the average annual contact allergy rates for the different preservatives are shown and are combined with the data from our earlier report (1). The numbers have been calculated by combining the positive patch test results for each preservative from each centre that submitted the data. Thus, for example, the data on paraben mix are based on 6123 patients for 2001 and on 5548 patients for the year 2005. The contact allergy rates for males versus females for the different

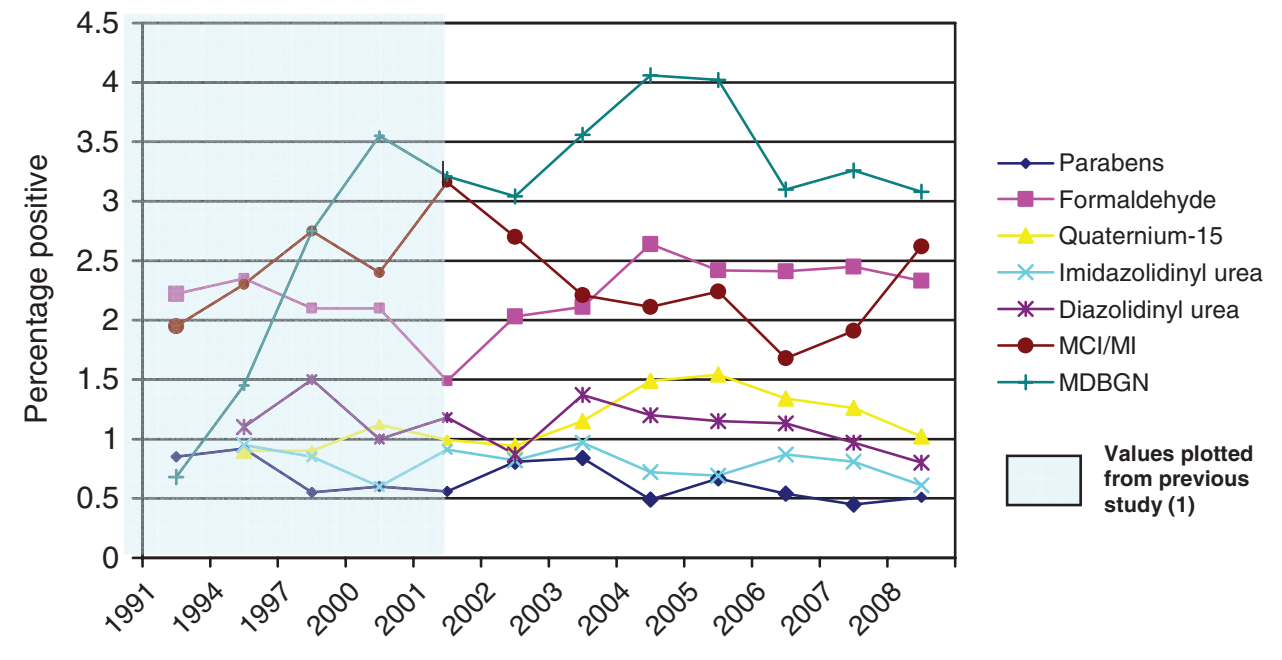

Fig. 1. The percentage of positive patch test reactions to preservatives averaged from data from the European centres in the period 2001-2008. MCI/MI, methylchloroisothiazolinone/methylisothiazolinone; MDBGN, methyldibromo glutaronitrile. 
preservatives studied differed, and no consistent patterns were found; hence, the results are not described here.

As in the previous publication(1), paraben mix remained the preservative with the lowest level of contact allergy, being in the range of $0.5-1 \%$ in consecutively tested patients referred with dermatitis. Also, the frequencies of contact sensitivity to quaternium-15, imidazolidinyl urea and diazolidinyl urea had not changed much from the previously reported frequencies. For formaldehyde, MDBGN, and MCI/MI, the frequencies of contact allergy stayed above $2 \%$ during the study period.

\section{Discussion}

MCI/MI contact allergy in the 1980 s led to recommendations to decrease the concentration recommended for use in leave-on and rinse-off products $(1,10)$. The nonhalogenated moiety MI was permitted by itself in 2005 , and it is now found in both industrial products and cosmetics (leave-on and rinse-off) $(11,12)$, which is leading to an increased rate of contact allergy to MI (11). In this article, only the MCI/MI rate is considered, because MI was not tested routinely in most clinics.

The total ban on MDBGN in cosmetics within the EU caused by the cases of contact allergy to MDBGN (13) has already led to a decrease in the occurrence of MDBGN allergy (14). The data in the current report cover the period of unrestricted use of the preservative to its prohibition in leave-on cosmetic products, but not to its total prohibition in all cosmetic products in Europe.

Formaldehyde caused problems in the 1960s that led to a decline in its use as a preservative/biocide, but there was an increase in the use of formaldehyde-releasers (11, 12, 15). It is well known that formaldehyde contact sensitivity may play a major role in positive reactions to formaldehyde-releasers $(15,16)$. However, formaldehyde contact sensitivity, which was reported with a steady frequency between $2 \%$ and $2.5 \%$ in the previous European study, now seems to have somewhat increased, being at a level of $\sim 2.5 \%$. The number is below those reported in Denmark, where an average of $3.1 \%$ was reported for the years 1985-2008 (2), and in other parts of the world both higher (17) and lower numbers have been mentioned $(3,18)$.

Legislation over the years can, with delay, be reflected in the levels of contact allergy to the preservatives/biocides that are affected by the legislation, and is a demonstration of the effectiveness of such interventions. Sensitization rates reflect the market use of preservatives in terms of the concentrations used and product types. The Dillarstone effect is a graphical representation of this (19).

Preservatives, because of their widespread use, continue to represent one of the major causes of contact allergy and this is shown in this pan-European follow-up.

\section{References}

1 Wilkinson J D, Shaw S, Andersen K E et al. Monitoring levels of preservative sensitivity in Europe. Contact Dermatitis 2002: 46: 207-210.

2 Thyssen J P, Engkilde K, Lundov M D et al. Temporal trends of preservative allergy in Denmark (1985-2008). Contact Dermatitis 2010: 62: 102-108.

3 Schnuch A, Lessman H, Geier J et al. Contact allergy to preservatives. Analysis of IVDK data 1996-2009. Br J Dermatol 2011: 164: 1316-1325.

4 Ozkaya E, Polat Ekinci A. Low sensitization rate but good clinical relevance of preservatives in Turkey: evaluation of 1256 patients over 10 years (1996-2006). J Dermatol 2011: 38 : 194-196.

5 Castelain M. Globalisation and allergy. Eur J Dermatol 2011: 21: 472-478.

6 Bruze M, Zimerson E. Dimethylfumarate. Dermatitis 2011: 22: 3-7.

7 Bruze M, Andersen K E, Goossens A. Recommendation to include fragrance mix 2 and hydroxyisohexyl 3-cyclohexene carboxaldehyde (Lyral) in the European baseline patch test series.
Contact Dermatitis 2008: 58: 129-133.

8 Bruze M, Conde-Salazar L, Goossens A et al. Thoughts on sensitizers in a standard patch test series. Contact Dermatitis 1999: 41: 241-250.

9 Andersen K E. Multicentre patch test studies: are they worth the effort? Contact Dermatitis 1998: 38: 222-223.

10 Cosmetic Ingredient Review Expert Panel of the Cosmetics Toiletry and Fragrance Association. Final report on the safety assessment of methylchloroisothiazolinone and methylisothiazolinone. J Am Coll Toxicol 1992: 11: 75-128.

11 Lundov M D, Johansen J D, Carlsen B C et al. Formaldehyde exposure and patterns of concomitant contact allergy to formaldehyde and formaldehyde-releasers. Contact Dermatitis 2010: 63: 31-36.

12 Flyvholm M A. Preservatives in registered chemical products. Contact Dermatitis 2005: 3: 27-32.

13 de Groot A C, de Cock P A, Coenraads P J et al. Methyldibromoglutaronitrile is an important contact allergen in The Netherlands. Contact Dermatitis 1996: 34: 118-120.

14 Johansen J D, Veien N, Laurberg G et al. Decreasing trends in methyldibromo glutaronitrile contact allergy following regulatory intervention. Contact Dermatitis 2008: 59: 48-51.

15 Steinberg D C. Voluntary registration of cosmetics and 2007 frequency preservative use. Cosmet Toiletries Mag 2008: 123: 47-52.

16 de Groot A C, Geier J, Flyvholm A-M et al. Formaldehyde-releasers: relationship to formaldehyde contact allergy, Part 2: metalworking fluids and remainder. Contact Dermatitis 2010: 63: 129-139.

17 Zug K A, Warshaw E M, Fowler J F Jr. et al. Patch-test results of the North American Contact Dermatitis Group 2005-2006. Dermatitis 2009: 20: 149-160.

18 Boyvat A, Akyol A, Gurgey E. Contact sensitivity to preservatives in Turkey. Contact Dermatitis 2005: 52: 329-332.

19 Dillarstone A. Cosmetic preservatives. Contact Dermatitis 1997: 37: 190. 